\begin{tabular}{|c|c|}
\hline Title & Hypoxic glucose metabolism in glioblastoma as a potential prognostic factor \\
\hline Author(s) & $\begin{array}{l}\text { Toy onaga, Takuya; Y amaguchi, Shigeru; Hirata, Kenji; Kobayashi, Kentaro; Manabe, Osamu; W atanabe, Shiro; } \\
\text { Terasaka, Shunsuke; Kobay ashi, Hiroyuki; Hattori, Naoya; Shiga, T ohru; Kuge, Y uji; T anaka, Shinya; Ito, Y oichi M.; } \\
\text { T amaki, Nagara }\end{array}$ \\
\hline Citation & $\begin{array}{l}\text { European Journal of Nuclear Medicine and Molecular Imaging, 44(4), 611-619 } \\
\text { https://doi.org/10.1007/s00259-016-3541-z }\end{array}$ \\
\hline Issue Date & 2017-04 \\
\hline Doc URL & http:/hdl.handle.net/2115/68666 \\
\hline Rights & The final publication is available at link.springer.com \\
\hline Tyре & article (author version) \\
\hline Additional Information & There are other files related to this item in HUSCAP. Check the above URL. \\
\hline File Information & EJNMMI44_611.pdf \\
\hline
\end{tabular}

Instructions for use 


\section{Hypoxic glucose metabolism in glioblastoma as a potential prognostic factor}

Takuya Toyonaga ${ }^{1}$, Shigeru Yamaguchi ${ }^{1,2}$, Kenji Hirata ${ }^{1}$, Kentaro Kobayashi ${ }^{3}$, Osamu Manabe $^{1}$, Shiro Watanabe ${ }^{1}$, Shunsuke Terasaka ${ }^{2}$, Hiroyuki Kobayashi ${ }^{2,}$ Naoya Hattori ${ }^{1}$, Tohru Shiga $^{1}$, Yuji Kuge ${ }^{4}$, Shinya Tanaka ${ }^{5}$, Yoichi M. Ito ${ }^{6}$, Nagara Tamaki ${ }^{1}$

${ }^{1}$ Department of Nuclear Medicine, Hokkaido University Graduate School of Medicine, Sapporo, Japan

${ }^{2}$ Department of Neurosurgery, Hokkaido University Graduate School of Medicine, Sapporo, Japan

${ }^{3}$ Department of Molecular Imaging, Hokkaido University Graduate School of Medicine, Sapporo, Japan

${ }^{4}$ Central Institute of Isotope Science, Hokkaido University, Sapporo, Japan

${ }^{5}$ Department of Cancer Pathology, Hokkaido University Graduate School of Medicine, Sapporo, Japan

${ }^{6}$ Department of Biostatistics, Hokkaido University Graduate School of Medicine, Sapporo, Japan

Corresponding author: Kenji Hirata, MD, $\mathrm{PhD}$

Assistant Professor

Department of Nuclear Medicine, Graduate School of Medicine, Hokkaido University Kita 15, Nishi 7, Kita-Ku, Sapporo, Hokkaido 060-8638, Japan

Tel: +81-11-706-5152; Fax: +81-11-706-7155

E-mail: khirata@med.hokudai.ac.jp 
Running Title: Hypoxic glucose metabolism in glioblastoma

Keywords: anaerobic glycolysis, glioblastoma, fluoromisonidazole, fluorodeoxyglucose, positron emission tomography 


\begin{abstract}
Purpose:

Metabolic activity and hypoxia are both important factors characterizing tumor aggressiveness. Here, we used F-18 fluoromisonidazole (FMISO) and F-18 fluorodeoxyglucose (FDG) positron emission tomography (PET) to define metabolically-active hypoxic volume, and investigate its clinical significance in relation to progression free survival (PFS) and overall survival (OS) in glioblastoma patients. Experimental Design:
\end{abstract}

Glioblastoma patients ( $\mathrm{n}=32$ ) underwent FMISO PET, FDG PET, and magnetic resonance imaging (MRI) before surgical intervention. FDG and FMISO PET images were coregistered with gadolinium-enhanced T1-weighted MR images. Volume of interest (VOI) of gross tumor volume (GTV) was manually created to enclose the entire gadolinium-positive areas. The FMISO tumor-to-normal region ratio (TNR) and FDG TNR were calculated in a voxel-by-voxel manner. For calculating TNR, standardized uptake value (SUV) was divided by averaged SUV of normal references. Contralateral frontal and parietal cortices were used as the reference region for FDG, whereas cerebellar cortex was used as the reference region for FMISO. FDG positive was defined as the FDG TNR $\geq 1.0$, and FMISO positive was defined as FMISO TNR $\geq 1.3$. Hypoxia volume (HV) was defined as the volume of FMISO positive, and metabolic tumor volume in hypoxia (hMTV) was the volume of FMISO/FDG double-positive. The total lesion glycolysis in hypoxia (hTLG) was hMTV $\times$ FDG SUVmean. The extent of resection (EOR) involving cytoreduction surgery was volumetric change based on planimetry methods using MRI. These factors were tested for correlation with patient prognosis. 
Results:

All tumor lesions were FMISO-positive and FDG-positive. Univariate analysis indicated that hMTV, hTLG, and EOR were significantly correlated with PFS ( $p=0.007$, $\mathrm{p}=0.04$, and $\mathrm{p}=0.01$, respectively) and that hMTV, hTLG, and EOR were also significantly correlated with $\mathrm{OS}(\mathrm{p}=0.0028, \mathrm{p}=0.037$, and $\mathrm{p}=0.014$, respectively). In contrast, none of FDG TNR, FMISO TNR, GTV, HV, patients' age, or Karnofsky Performance Scale (KPS) was significantly correlated with PSF or OS. The hMTV and hTLG were found to be independent factors affecting PFS and OS on multivariate analysis.

Conclusions:

We introduced hMTV and hTLG using FDG and FMISO PET to define metabolically-active hypoxic volume. Univariate and multivariate analyses demonstrated that both hMTV and hTLG are significant predictors for PFS and OS in glioblastoma patients.

\section{Introduction}

Glioblastoma is the most aggressive astrocytic tumor among cerebral gliomas. It is defined as grade IV according to the 2007 World Health Organization (WHO) classification [1]. Recently, the prognosis of glioblastoma patients has been significantly improved owing to the development of image-guided surgery and radiotherapy and new chemotherapeutic drugs; however, the 2-year survival rate remains at only $27 \%$ [2].

Since glioblastomas frequently develop severe hypoxia [3-8], the most important pathological characteristics that discriminate glioblastoma from lower grade 
gliomas are necrosis and microvascular proliferation [1]. F-18 fluoromisonidazole (FMISO) positron emission tomography (PET) is the most widely used technique for the localization and quantification of hypoxia in vivo [9], and enables the non-invasive detection of hypoxia in glioblastoma patients [5].

Tumor cells can adapt themselves to hypoxia by promoting the genes which drive glucose metabolism and allow the tumor cells to survive and proliferate in hypoxia. In such conditions, glucose is metabolized without consuming oxygen to produce lactate (anaerobic glycolysis) whereas oxygen is essential to fully metabolize glucose (oxidative phosphorylation) [10]. Hypoxia imaging has been reported to provide prognostic information in glioblastoma patients; for example, previous studies found that glioblastoma patients with a larger hypoxia volume (HV) in the tumor had shorter survival [11-14]. However, hypoxia imaging may reflect not only active tumor cells but also dying cells that could not survive severe hypoxic conditions [8].

Metabolic activity can be measured using F-18 fluorodeoxyglucose (FDG) PET. According to previous reports, both the intensity and volume of glucose metabolism was associated with poor prognosis in glioma patients [15-17]. Thus, metabolically active HV can be segmented as the volume showing both FMISO and FDG uptakes. Additionally, because FMISO accumulates in severely hypoxic tissue $(\leq 10 \mathrm{mmHg})$ where normal neurons and glial cells cannot survive, we hypothesized that FDG within FMISO-positive areas all represent tumor metabolism. In the present study, we aimed to investigate the volume of anaerobic glycolysis in relation to patient outcome.

\section{Materials and Methods}




\section{Study subjects}

The Ethics Committee of Hokkaido University Hospital approved this study. Written informed consent was obtained from each patient who participated in the study. Using electronic medical records we reviewed a total of 176 FMISO PET scans from 141 patients for the investigation of possible brain tumors. Patients who did not undergo surgery (resection or biopsy) were excluded. Considering the possible modification of metabolism or hypoxia status by interventions, we further excluded patients who had already received any treatments before PET scanning, including anti-cancer chemotherapy, radiotherapy, and tumor resection. Among them, 32 patients were diagnosed as having glioblastoma. The final study population consisted of 17 male and 15 female glioblastoma patients (age, $63.5 \pm 14.8$ years). The patients’ characteristics’ are shown in Table 1. Pathological diagnosis was determined by agreement between two experienced neuropathologists based on the WHO 2007 classification [1]. All of the 32 glioblastoma patients received additional chemoradiotherapy with temozolomide.

\section{Image acquisition and reconstruction}

Magnetic resonance imaging (MRI) was conducted to generate the following image series: T1-weighted images with and without gadolinium contrast medium, T2-weighted images, and fluid-attenuated inversion recovery (FLAIR) images. FDG and FMISO PET were conducted in random order with an interval of $<7$ days. Both FDG and FMISO PET protocols were the same as in our previous study, with the exception of the types of scanner used [5]. Briefly, for FMISO PET, static PET scanning was initiated at 4 hours after intravenous injection. The actual interval and dosage were 
4.06 \pm 0.30 hours, and $413.9 \pm 28.2 \mathrm{MBq}$, respectively.

We used one of three PET scanners: an ECAT EXACT HR+ PET scanner (Asahi-Siemens Medical Technologies Ltd., Tokyo, Japan), a Biograph 64 PET computed tomography (CT) scanner (Asahi-Siemens Medical Technologies Ltd., Tokyo, Japan), and Gemini TF64 PET-CT scanner (Hitachi Medical Corporation Ltd, Tokyo, Japan). All scanners were operated in three-dimensional mode. In the case ofthe ECAT EXACT HR+ PET scanner, the 10-minute emission acquisition was followed by a 3-minute transmission scan with a ${ }^{68} \mathrm{Ge} /{ }^{68} \mathrm{Ga}$ retractable line source. In the case of the Biograph 64 PET/CT and Gemini TF64 PET-CT scanners, CT scanning for attenuation correction was followed by a 10-minute emission acquisition. Attenuation-corrected radioactivity images were reconstructed using filtered back projection (ECAT EXACT HR+ and Biograph 64) or the ordered subset expectation maximization method (Gemini TF64) with a Hann filter of 4 mm full width at half maximum.

On the day of the FDG PET scan, the patients fasted for $>6$ hours before the study. The static PET images were acquired at 1 hour after intravenous FDG (4.5MBq/kg) injection. The actual interval and dosage were $1.23 \pm 0.30$ hours, and 323.3 $\pm 90.6 \mathrm{MBq}$, respectively. The other imaging protocols for FDG, including acquisition time and image reconstruction, were the same as that for FMISO.

\section{Image analysis}

Definition of volume of interest: FDG and FMISO PET images, and FLAIR and gadolinium-enhanced T1-weighted MR images were coregistered with individual gadolinium-enhanced T1-weighted MR images using SPM8 software [18]. A nuclear medicine physician created the volumes of interest (VOIs) manually to enclose the 
entire area showing gadolinium enhancement as the gross tumor volume (GTV), and to enclose the entire area exhibiting a high intensity of FLAIR in the tumor lesion. Every VOI was created using an application tool that was developed in our institute. Polygonal regions of interest (ROIs) were drawn on all the slices that showed gadolinium enhancement of the tumor. The boundaries of the ROIs were made 3-5 mm outside of the Gd enhancement area to include all of the gadolinium-enhanced voxels in the ROIs. The VOI was generated by integrating all of the polygonal ROIs as the tumor lesion.

The normal reference regions were identified on the gadolinium-enhanced T1-weighted MR images of each patient. Contralateral frontal and parietal cortices were used as the reference region in the case of FDG, whereas cerebellar cortex was used as the reference region in the case of FMISO. Circular ROIs (10 $\mathrm{mm}$ in diameter) were placed in the manner described as follows.1) For FMISO, we placed 30 ROIs on the cerebellar cortex (5 ROIs on each hemisphere per slice $\times 3$ axial slices $\times 2$ sides). The averaged value was used for the reference. 2) For FDG, we placed six ROIs on each of the three axial slices on the contralateral frontoparietal cortex. The averaged value was used for the reference. Another nuclear medicine physician visually confirmed that all tumoral and normal ROIs were defined appropriately. Both nuclear medicine physicians had completed neuroradiology training in our institute. The tumor-to-normal region ratio (TNR) was calculated by dividing the maximum standardized uptake value (SUV) of the tumor lesions by the averaged SUV of normal reference. For FMISO, we averaged cerebellar uptake for the normal reference, because use of target-to-cerebellum ratio has been recommended to most accurately approximate distribution volume [19]. For FDG reference, cerebellum is not appropriate because the cerebellum cortex uptake is often reduced by contralateral supratentorial tumor (so called crossed cerebellar 
diaschisis or CCD). Instead, contralateral cortex, which we used in this study, has been commonly adopted for FDG normal reference.

Volume definition: Within the VOIs with FLAIR high intensity, a voxel that had an FMISO TNR $\geq 1.3$ was defined as FMISO-positive; this was because we successfully detected tumor hypoxia in our previous study[5]. The FMISO-positive region was considered as the HV. The voxel having an FDG TNR $\geq 1.0$ was defined as FDG-positive. Metabolic tumor volume (MTV) measurement for brain tumor is interfered by physiological FDG uptake in the surrounding brain tissues. Thus, here we propose a new parameter, namely hypoxic MTV (hMTV), which is the volume showing as FMISO-positive and FDG-positive (Figure 1). Here, we define hypoxic total lesion glycolysis (hTLG) as [hMTV]×[SUVmean within hMTV]. The hypermetabolic fraction of hypoxic volume (HMF) was defined as [hMTV]/[HV]. The GTV, HV, hMTV, hTLG, and HMF were examined in relation to patient outcome.

\section{Extent of resection}

The extent of resections (EOR) using cytoreduction surgery was quantitatively estimated by volumetric change based on planimetry methods using a conventional 67-mm-thick slice of Gd-T1WI MRI as described previously[20]. Pre- and post-operative tumor volumes were calculated as the sum of the Gd-enhanced area that was determined manually; then the resected tumor volume was determined by subtraction of the post-operative tumor volume from the pre-operative tumor volume. The EOR was defined as the percentage of resected tumor volume in the pre-operative tumor volume. Post-operative tumor volumes were assessed using Gd-T1WI MRI that was performed 
at $<48$ hours after surgery to avoid post-operative changes showing Gd enhancement. In needle biopsy cases, the EOR were defined as $0 \%$.

\section{Surgical procedures}

The surgical procedure was performed in the same manner as described previously [21, 22]. We used an intraoperative neuronavigation system (Stealth Station TREON: Medtronic, Louisville, CO, USA). Gadolinium-enhanced T1-weighted imaging (Gd-T1WI) with three-dimensional spoiled gradient echo (3D-SPGR) MR images and PET images were transferred to the navigation system. FDG PET was used to identify the most active tumor tissue. The surgical procedure was either biopsy or tumor resection. We attempted to remove as much tumor tissue as possible in every case. However, we experienced patients with tumors confined to deep brain structures (e.g., the thalamus) or involving eloquent brain areas (e.g., the perisylvian verbal area) or both. These patients received only biopsy for histological diagnosis because they were not feasible candidates for cytoreductive surgery. In the biopsy cases, following a small craniotomy under general anesthesia, a needle biopsy probe was inserted at a target point within the region with the highest uptake of the tracer. After this, the probe was fixed at the target point, and up to five tissue samples were aspirated through the probe. With the exception of such biopsy cases, we performed a maximum safe resection of the regions of the lesion that exhibited higher tracer uptake than the surrounding brain parenchyma. At least, the tissue showing the highest tracer uptake was carefully

resected. To determine the resection boundary, three or four silicon catheters were inserted around the targeted lesion under the guidance of a navigation system before dural opening to avoid inaccuracy caused by brain shift [23]. After insertion of these 
catheter tubes, we performed a dural incision and resected as much tumor tissue as possible under the guidance of the catheter tubes, as well as other neurophysiological monitoring.

In total, 19, 6, and 7 patients underwent sub-total or gross-total resection $(\mathrm{EOR}=90.3-100 \%)$, partial resection $(\mathrm{EOR}=10.6-85.5 \%)$, and biopsy $(\mathrm{EOR}=0-2.1 \%)$, respectively.

\section{Prognosis}

Overall survival (OS) was defined as the time period (months) between the date of surgery and the date of death for any reason. Progression-free survival (PFS) was defined as the time period (months) between the date of surgery and the date that tumor recurrence was confirmed using any imaging modalities. We observed all patients for $>2$ months or until death. Twenty-four patients experienced tumor recurrence, 20 of whom died during the follow-up period (OS=3.2-46.8 months). The remaining 8 patients without recurrence were all alive at the end of the follow-up period, resulting in a total of 12 patients being alive at the end of the follow-up period (2.7-45.1 months).

\section{Statistical analysis}

All continuous variables are presented as the mean \pm standard deviation. In this study, we attempted to exhaustively determine the optimal cutoff values for each parameter to best categorize patients by OS. We repeatedly applied the log-rank test to each parameter and decided the optimal cutoff values that showed maximum $\chi^{2}$ statistics. This method was applied to the TNR, GTV, HV, hMTV, hTLG, HMF, EOR, 
Karnofsky performance status and patient age. The survival curves were created using the Kaplan-Meier method. We tested the correlation between PFS or OS and parameters using the Cox regression model for univariate analysis and with the Cox proportional hazard model for multivariate analysis. To avoid a problem of multiple testing, the $\mathrm{p}$ values of univariate analyses were corrected by the method which was previously reported [24].

All the statistical analyses were performed using R version 3.1.2 and 'survival' add-on package [25]. P values of $<0.05$ were considered statistically significant.

\section{Results}

\section{SUVmax and tumor volumes}

The SUVmax for FDG was $8.44 \pm 3.00$ (range, 4.19-15.03), the TNR for FDG was $1.55 \pm 0.52$ (range, 1.08-3.96). All (100\%) of the lesions had a higher FDG SUVmax than the reference value (i.e., TNR $\geq 1.0$ ), and thus were considered to have a hypermetabolic volume in the tumor. The SUVmax for FMISO was $3.75 \pm 1.30$ (range, 1.71-6.91), and the TNR for FMISO was 3.29 \pm 0.81 (range, 1.48-4.85). In volumetric assessment, the hMTV and HV were $7.98 \pm 8.85 \mathrm{ml}$ (range, $0.12-35.27 \mathrm{ml}$ ) and 29.78 \pm 18.90 (range, $0.84-81.95 \mathrm{ml}$ ), respectively. The hTLG was $41.69 \pm 37.65$ (range, 0.66-126.26), and the HMF was $25.15 \pm 18.03 \%$ (range, 0.89-61.80\%).

\section{Univariate analysis}

Table2 summarizes the optimal cutoff values that generated maximum $\chi^{2}$ 
statistics for each parameter in categorizing patients by OS. At these cutoff values, the hMTV, hTLG, and EOR were significantly correlated with PFS ( $p=0.007, p=0.04$, and $\mathrm{p}=0.01$, respectively) using univariate analysis. A significantly shorter OS was also suggested by higher HV, higher hMTV, higher hTLG, and lower EOR $(p=0.04$, $\mathrm{p}=0.0028, \mathrm{p}=0.037$, and $\mathrm{p}=0.014$, respectively) (Table 3). Seven patients with a higher hMTV (i.e., hMTV $\geq 15.01$ ) had a significantly shorter OS than the remaining 25 patients with a lower hMTV (i.e., hMTV <15.01); the median OS was 5.3 months for the high hMTV group (n=7) vs. 15.7 months for the low hMTV group (n=25) (Figure 2A). Twelve patients with a higher hTLG (i.e., hTLG $\geq 49.41$ ) had a significantly shorter OS than the remaining 20 patients with a lower hTLG (i.e., hTLG <49.41); the median OS was 5.8 months for the high hTLG group $(n=12)$ vs. 15.7 months for the low hTLG group ( $n=20$ ) (Figure 2B). Sixteen patients with a higher EOR (i.e., EOR $\geq 94.5 \%$ ) had a significantly longer OS than those with a lower EOR (i.e., EOR <94.5\%); the median OS was 21.6 months for the low-EOR group ( $\mathrm{n}=16)$ vs. 11.3 months for the low-EOR group ( $\mathrm{n}=16)$ in common with previous reports (Figure 2C). The Kaplan-Meier analysis using medians of hMTV and hTLG as the thresholds did not reach statistical significance (supplementary document).

\section{Multivariate analysis}

Because the hMTV and hTLG were significantly correlated (Pearson’s R=0.90; $\mathrm{p}<0.0001$ ), we constructed two separate models to avoid the co-linearity problem. Model 1 consisted of the hMTV + HV + EOR, and model 2 consisted of the hTLG + HV + EOR. The Cox proportional hazard model for hMTV, HV, and EOR used to predict OS (model 1) indicated that the hMTV and EOR were independent prognostic 
factors ( $\mathrm{p}=0.0063$ and $\mathrm{p}=0.013$, respectively; Table 3 ). Analysis using model 2 similarly suggested that the hTLG and EOR independently affected OS ( $p=0.011$ for hTLG and $\mathrm{p}=0.0042$ for EOR; Table 3). PFS was also independently influenced by the hMTV or hTLG (Table 4).

\section{Discussion}

We conducted a study involving the independent detection of hypermetabolic and hypoxic volumes using FDG PET and FMISO PET, respectively. The parameters hMTV and hTLG were tested as prognostic factors. Using univariate analysis we found that the patients who had a larger hMTV or larger hTLG, or had undergone a more extensive surgical procedure, survived significantly longer than the other patients. In addition, multivariate analysis indicated that both the hTLG and hMTV were significant prognostic factors that were independent from the EOR.

Recent studies have reported that a lower EOR was a predictor of poor prognosis in glioma patients [26-32]. Our study confirmed this finding. In addition to the EOR, we newly found that the hTLG and hMTV may predict patient prognosis. These prognostic factors might be independent from the EOR, while further studies will be needed to investigate a large number of patients from multiple institutes before confirming the current results. The point is that we can determine these parameters during the pre-treatment phase. These findings may provide the necessary information for the detection of the patients who will develop early recurrence regardless of the surgical procedure. Such patients might be considered eligible for additional treatment in the future, such as expanding resection of the non-eloquent area, radiation dose 
escalation, hypoxia-activated chemotherapy, immunotherapy, or photodynamic therapy.

In malignant tumor cells, especially under severely hypoxic conditions, HIF-1 $\alpha$ is often promoted through signal transductional pathways, such as the phosphoinositide-3-kinase/Akt/mTOR pathway, extracellular regulated protein kinase pathway, and adenosine 5'-monophosphate activated protein kinase pathway. HIF-1 $\alpha$ forms a complex with HIF-1 $\beta$ or with other proteins, and adheres to hypoxia responsive elements (HRE; i.e., 5'-ACGTG-3') on the genome line. HRE stimulates the downstream enzymes in the glycolytic system such as glucose transporters, hexokinase, pyruvate kinase, and lactate dehydrogenase A [33]. This cascade helps tumor cells metabolize glucose anaerobically and survive under severe hypoxia, the so called Warburg effect.

In the present study, we hypothesized that FDG uptake intensity in the hypoxic area (i.e., the FMISO-positive area) could represent the tumor's ability to adapt to severe hypoxia. In other words, the FDG/FMISO double-positive fraction may reflect 'signal transmission efficiency' initiated from hypoxia to anaerobic glycolysis. Therefore, we investigated the intensity of anaerobic glycolysis by using the new parameters hMTV and hTLG.

According to previous reports, both the intensity and volume of glucose metabolism were associated with poor prognosis in glioma patients [15-17]. FDG is a very good tracer for evaluating tumor metabolic activity. It may be argued that all that is needed is FDG PET for the purpose of measuring active tumor volume. In non-brain malignancies, FDG PET has been recently analyzed using volume-based parameters such as MTV and TLG [34]. These parameters, however, are difficult to measure for brain tumors because of the physiological uptake of FDG in the brain. Because FMISO 
uptake reflects severe hypoxia $(<10-20 \mathrm{mmHg})$, normal brain tissues would not be able to survive within a FMISO-positive region. Therefore, the FDG uptake within a FMISO-positive region should exclusively reflect tumoral glucose metabolism, and thus the volume of FDG/FMISO double-positive lesions would reasonably reflect viable tumor volume. ${ }^{11} \mathrm{C}$-methionine PET is a well-known technique that can more accurately reflect the tumor volume than MRI [35]. We have recently reported that ${ }^{11} \mathrm{C}$-methionine uptake volume is a good parameter for the prediction of prognosis in high-grade glioma [22]. The current study is in line with that study in terms of the clinical significance of viable tumor volume.

The current data are not enough to determine which parameter is the more reliable and the more robust predictor between hMTV and hTLG. The two parameters were strongly correlated (Pearson's $\mathrm{R}=0.90 ; \mathrm{p}<0.0001$ ). Previous studies in head-and-neck cancer patients showed that both MTV and TLG were significant predictors [36-40], and some of them show the superiority of TLG [36, 39]. Because TLG represents the total amount of FDG taken up in the tumor lesion (i.e., mathematically, TLG is the integral of FDG SUV within the tumor boundary), TLG might reflect both tumor cell number and malignancy per cell.

Previous studies reported the correlation between the $\mathrm{HV}$ and prognosis of glioma patients $[11,14]$. Hypoxia is well known to induce radiation resistance, because free radical-induced DNA damage requires oxygen molecules [41]. Lactate is produced by anaerobic glycolysis and may scavenge superoxide and hydroxyl radicals [42]. Hypoxia also induces resistance to many type of chemotherapy. Temozolomide, which is the main treatment agent against high-grade glioma, reduces its treatment effect under hypoxia [43]. It is reasonable that tumor hypoxia causes poor prognosis by means of 
such treatment resistance.

However, in our current study, HV was not a significant independent prognostic factor. We may need further investigation because there are differences between our study and previous studies, including FMISO delineating threshold and the waiting time after FMISO injection. The size of our study population was relatively small. One of the possible reasons may be the biological heterogeneity inside the FMISO-positive area. The specimens from the FMISO-positive area have been reported to contain necrotic tissue, dying cells, and viable cells [8]. There might be a relationship between necrosis and FMISO uptake; however, FMISO is not taken up in necrotic tissues themselves. Instead, FMISO may accumulate in peri-necrotic viable tumor cells where oxygen concentration is not satisfactory. Theoretically, tumor aggressiveness should be ascribed to the viable cell fraction but not to necrotic tissue or dying cells. Thus, we introduced new parameters from this view point. The new parameters that reflected FDG intensity in FMISO-positive areas could represent the metabolic activity of viable cells under hypoxia. Because the patients with larger hMTV and hTLG may have more viable cells under hypoxia, they have greater possibility of recurrence regardless of successful maximum safe resection. Use of hypoxia imaging has been reported for the planning of radiotherapy [44], diagnosing glioma [5], or voxel-by-voxel analysis with FDG PET [45]. However, to our knowledge, the current report is the first to introduce anaerobic glycolytic activity as a prognostic factor.

We exhaustively analyzed the patients' prognosis using every possible cutoff value and determined the optimal threshold using an established method [24]. Although exhaustive analysis contained median and average, those thresholds failed to differentiate patients between better and poor prognosis, partly due to skewed 
distribution of PET parameters.

We need to mention the limitations regarding the study. First, the number of patients enrolled was small. Although survival analysis was limited, the number of variables making up the multivariate models was reasonable; this was confirmed by a co-author who is an experienced statistician (Y.I.). Second, the PET images investigated were acquired using several types of PET scanner. The differences in spatial resolution and image contrast may have affected delineation of the FDG and FMISO positive lesions. However, to minimize these effects, the images were reconstructed using appropriate smoothing filters to match the full width at half maximum of the reconstructed images with each other.

\section{Conclusion}

We introduced the parameters hMTV and hTLG using FDG PET and FMISO PET for glioblastoma patients for the semi-quantitative estimation of anaerobic glucose metabolism. Univariate and multivariate analyses demonstrated that both hMTV and hTLG are significant predictors for OS in glioblastoma patients.

\section{Compliance with Ethical Standards}

The authors declare that they have no conflict of interest. All procedures performed in studies involving human participants were in accordance with the ethical standards of the institutional and/or national research committee and with the 1964 Helsinki declaration and its later amendments or comparable ethical standards. Informed consent was obtained from all individual participants included in the study. 


\section{Financial Disclosure}

This work was supported in part by the Creation of Innovation Centers for Advanced Interdisciplinary Research Areas Program, Ministry of Education, Culture, Sports, Science and Technology, Japan, from the SNMMI Wagner-Torizuka Fellowship from 2013 to 2015 (to Kenji Hirata), by the Hokkaido University HIROKO’s Fund for Academic Exchange from 2012 to 2014 (to Kenji Hirata), and by a research grant from the Japan Radiological Society from Bayer from April 2015 to March 2016 (to Kenji Hirata). The funders had no role in study design, data collection and analysis, decision to publish, or preparation of the manuscript.

The authors have no conflict of interest to disclose with respect to this manuscript.

\section{References}

1. Louis DN, Ohgaki H, Wiestler OD, Cavenee WK, Burger PC, Jouvet A et al. The 2007 WHO classification of tumours of the central nervous system. Acta neuropathologica. 2007;114(2):97-109. doi:10.1007/s00401-007-0243-4.

2. Omuro A, DeAngelis LM. Glioblastoma and other malignant gliomas: a clinical review. Jama. 2013;310(17):1842-50. doi:10.1001/jama.2013.280319.

3. Flynn JR, Wang L, Gillespie DL, Stoddard GJ, Reid JK, Owens J et al. Hypoxia-regulated protein expression, patient characteristics, and preoperative imaging as predictors of survival in adults with glioblastoma multiforme. Cancer. 2008;113(5):1032-42. doi:10.1002/cncr.23678. 
4. Oliver L, Olivier C, Marhuenda FB, Campone M, Vallette FM. Hypoxia and the malignant glioma microenvironment: regulation and implications for therapy. Current molecular pharmacology. 2009;2(3):263-84.

5. Hirata K, Terasaka S, Shiga T, Hattori N, Magota K, Kobayashi H et al. (1)(8)F-Fluoromisonidazole positron emission tomography may differentiate glioblastoma multiforme from less malignant gliomas. European journal of nuclear medicine and molecular imaging. 2012;39(5):760-70. doi:10.1007/s00259-011-2037-0. 6. Evans SM, Judy KD, Dunphy I, Jenkins WT, Hwang WT, Nelson PT et al. Hypoxia is important in the biology and aggression of human glial brain tumors. Clinical cancer research : an official journal of the American Association for Cancer Research. 2004;10(24):8177-84. doi:10.1158/1078-0432.CCR-04-1081.

7. Lally BE, Rockwell S, Fischer DB, Collingridge DR, Piepmeier JM, Knisely JP. The interactions of polarographic measurements of oxygen tension and histological grade in human glioma. Cancer journal. 2006;12(6):461-6.

8. Toyonaga T, Hirata K, Yamaguchi S, Hatanaka KC, Yuzawa S, Manabe O et al. F-fluoromisonidazole positron emission tomography can predict pathological necrosis of brain tumors. European journal of nuclear medicine and molecular imaging. 2016. doi:10.1007/s00259-016-3320-х. 
9. Bell C, Dowson N, Fay M, Thomas P, Puttick S, Gal Y et al. Hypoxia imaging in gliomas with 18F-fluoromisonidazole PET: toward clinical translation. Seminars in nuclear medicine. 2015;45(2):136-50. doi:10.1053/j.semnuclmed.2014.10.001.

10. Jensen RL. Brain tumor hypoxia: tumorigenesis, angiogenesis, imaging, pseudoprogression, and as a therapeutic target. Journal of neuro-oncology. 2009;92(3):317-35. doi:10.1007/s11060-009-9827-2.

11. Spence AM, Muzi M, Swanson KR, O'Sullivan F, Rockhill JK, Rajendran JG et al. Regional hypoxia in glioblastoma multiforme quantified with [18F]fluoromisonidazole positron emission tomography before radiotherapy: correlation with time to progression and survival. Clinical cancer research : an official journal of the American Association for Cancer Research. 2008;14(9):2623-30. doi:10.1158/1078-0432.CCR-07-4995. 12. Swanson KR, Chakraborty G, Wang CH, Rockne R, Harpold HL, Muzi M et al. Complementary but distinct roles for MRI and 18F-fluoromisonidazole PET in the assessment of human glioblastomas. Journal of nuclear medicine : official publication, Society of Nuclear Medicine. 2009;50(1):36-44. doi:10.2967/jnumed.108.055467. 13. Szeto MD, Chakraborty G, Hadley J, Rockne R, Muzi M, Alvord EC, Jr. et al. Quantitative metrics of net proliferation and invasion link biological aggressiveness assessed by MRI with hypoxia assessed by FMISO-PET in newly diagnosed 
glioblastomas. Cancer research. 2009;69(10):4502-9.

doi:10.1158/0008-5472.CAN-08-3884.

14. Kawai N, Lin W, Cao WD, Ogawa D, Miyake K, Haba R et al. Correlation between (1)(8)F-fluoromisonidazole PET and expression of HIF-1alpha and VEGF in newly diagnosed and recurrent malignant gliomas. European journal of nuclear medicine and molecular imaging. 2014;41(10):1870-8. doi:10.1007/s00259-014-2776-9.

15. Colavolpe C, Metellus P, Mancini J, Barrie M, Bequet-Boucard C, Figarella-Branger D et al. Independent prognostic value of pre-treatment 18-FDG-PET in high-grade gliomas. Journal of neuro-oncology. 2012;107(3):527-35. doi:10.1007/s11060-011-0771-6. 16. Spence AM, Muzi M, Graham MM, O'Sullivan F, Link JM, Lewellen TK et al. 2-[(18)F]Fluoro-2-deoxyglucose and glucose uptake in malignant gliomas before and after radiotherapy: correlation with outcome. Clinical cancer research : an official journal of the American Association for Cancer Research. 2002;8(4):971-9. 17. Pardo FS, Aronen HJ, Fitzek M, Kennedy DN, Efird J, Rosen BR et al. Correlation of FDG-PET interpretation with survival in a cohort of glioma patients. Anticancer research. 2004;24(4):2359-65.

18. Friston KJ, Holmes, A.P., Worsley, K.J., Poline, J.P., Frith, C.D., Frackowiak, R.S.J. 
Statistical parametric maps in functional imaging: a general linear approach. Human Brain Mapping. 1995.

19. Bruehlmeier M, Roelcke U, Schubiger PA, Ametamey SM. Assessment of hypoxia and perfusion in human brain tumors using PET with 18F-fluoromisonidazole and 15O-H2O. Journal of nuclear medicine : official publication, Society of Nuclear Medicine. 2004;45(11):1851-9.

20. Yamaguchi S, Kobayashi H, Terasaka S, Ishii N, Ikeda J, Kanno H et al. The impact of extent of resection and histological subtype on the outcome of adult patients with high-grade gliomas. Japanese journal of clinical oncology. 2012;42(4):270-7. doi:10.1093/jjco/hys016.

21. Yamaguchi S, Kobayashi H, Hirata K, Shiga T, Tanaka S, Murata J et al. Detection of histological anaplasia in gliomas with oligodendroglial components using positron emission tomography with (18)F-FDG and (11)C-methionine: report of two cases. Journal of neuro-oncology. 2011;101(2):335-41. doi:10.1007/s11060-010-0262-1. 22. Kobayashi K, Hirata K, Yamaguchi S, Manabe O, Terasaka S, Kobayashi H et al. Prognostic value of volume-based measurements on (11)C-methionine PET in glioma patients. European journal of nuclear medicine and molecular imaging. 2015;42(7):1071-80. doi:10.1007/s00259-015-3046-1. 
23. Yoshikawa K, Kajiwara K, Morioka J, Fujii M, Tanaka N, Fujisawa H et al.

Improvement of functional outcome after radical surgery in glioblastoma patients: the efficacy of a navigation-guided fence-post procedure and neurophysiological monitoring. Journal of neuro-oncology. 2006;78(1):91-7.

doi:10.1007/s11060-005-9064-2.

24. Altman DG, Lausen B, Sauerbrei W, Schumacher M. Dangers of using "optimal" cutpoints in the evaluation of prognostic factors. Journal of the National Cancer Institute. 1994;86(11):829-35.

25. Terry M Therneau TL. A Packagefor Survival Analysis in S. 2014.

26. Sanai N, Polley MY, McDermott MW, Parsa AT, Berger MS. An extent of resection threshold for newly diagnosed glioblastomas. Journal of neurosurgery. 2011;115(1):3-8. doi:10.3171/2011.2.JNS10998 10.3171/2011.7.JNS10238.

27. Pope WB, Sayre J, Perlina A, Villablanca JP, Mischel PS, Cloughesy TF. MR imaging correlates of survival in patients with high-grade gliomas. AJNR American journal of neuroradiology. 2005;26(10):2466-74.

28. Keles GE, Anderson B, Berger MS. The effect of extent of resection on time to tumor progression and survival in patients with glioblastoma multiforme of the cerebral 
hemisphere. Surgical neurology. 1999;52(4):371-9.

29. Sanai N, Berger MS. Glioma extent of resection and its impact on patient outcome. Neurosurgery. 2008;62(4):753-64; discussion 264-6.

doi:10.1227/01.neu.0000318159.21731.cf.

30. Lacroix M, Abi-Said D, Fourney DR, Gokaslan ZL, Shi W, DeMonte F et al. A multivariate analysis of 416 patients with glioblastoma multiforme: prognosis, extent of resection, and survival. Journal of neurosurgery. 2001;95(2):190-8.

doi:10.3171/jns.2001.95.2.0190.

31. Hardesty DA, Sanai N. The value of glioma extent of resection in the modern neurosurgical era. Frontiers in neurology. 2012;3:140. doi:10.3389/fneur.2012.00140. 32. Marko NF, Weil RJ, Schroeder JL, Lang FF, Suki D, Sawaya RE. Extent of resection of glioblastoma revisited: personalized survival modeling facilitates more accurate survival prediction and supports a maximum-safe-resection approach to surgery. Journal of clinical oncology : official journal of the American Society of Clinical Oncology. 2014;32(8):774-82. doi:10.1200/JCO.2013.51.8886.

33. Gao JL, Chen YG. Natural Compounds Regulate Glycolysis in Hypoxic Tumor Microenvironment. BioMed research international. 2015;2015:354143. doi:10.1155/2015/354143. 
34. Pak K, Cheon GJ, Nam HY, Kim SJ, Kang KW, Chung JK et al. Prognostic value of metabolic tumor volume and total lesion glycolysis in head and neck cancer: a systematic review and meta-analysis. Journal of nuclear medicine : official publication, Society of Nuclear Medicine. 2014;55(6):884-90. doi:10.2967/jnumed.113.133801. 35. Terakawa Y, Tsuyuguchi N, Iwai Y, Yamanaka K, Higashiyama S, Takami T et al. Diagnostic accuracy of 11C-methionine PET for differentiation of recurrent brain tumors from radiation necrosis after radiotherapy. Journal of nuclear medicine : official publication, Society of Nuclear Medicine. 2008;49(5):694-9. doi:10.2967/jnumed.107.048082.

36. Suzuki H, Nishio M, Nakanishi H, Hanai N, Hirakawa H, Kodaira T et al. Impact of total lesion glycolysis measured by 18F-FDG-PET/CT on overall survival and distant metastasis in hypopharyngeal cancer. Oncology letters. 2016;12(2):1493-500. doi:10.3892/ol.2016.4765.

37. Abd El-Hafez YG, Moustafa HM, Khalil HF, Liao CT, Yen TC. Total lesion glycolysis: a possible new prognostic parameter in oral cavity squamous cell carcinoma. Oral oncology. 2013;49(3):261-8. doi:10.1016/j.oraloncology.2012.09.005. 38. Lim R, Eaton A, Lee NY, Setton J, Ohri N, Rao S et al. 18F-FDG PET/CT metabolic tumor volume and total lesion glycolysis predict outcome in oropharyngeal squamous 
cell carcinoma. Journal of nuclear medicine : official publication, Society of Nuclear Medicine. 2012;53(10):1506-13. doi:10.2967/jnumed.111.101402.

39. Moon SH, Choi JY, Lee HJ, Son YI, Baek CH, Ahn YC et al. Prognostic value of 18F-FDG PET/CT in patients with squamous cell carcinoma of the tonsil: comparisons of volume-based metabolic parameters. Head \& neck. 2013;35(1):15-22. doi:10.1002/hed.22904.

40. Picchio M, Kirienko M, Mapelli P, Dell'Oca I, Villa E, Gallivanone F et al. Predictive value of pre-therapy (18)F-FDG PET/CT for the outcome of (18)F-FDG PET-guided radiotherapy in patients with head and neck cancer. European journal of nuclear medicine and molecular imaging. 2014;41(1):21-31. doi:10.1007/s00259-013-2528-2.

41. Meijer TW, Kaanders JH, Span PN, Bussink J. Targeting hypoxia, HIF-1, and tumor glucose metabolism to improve radiotherapy efficacy. Clinical cancer research : an official journal of the American Association for Cancer Research. 2012;18(20):5585-94. doi:10.1158/1078-0432.CCR-12-0858.

42. Groussard C, Morel I, Chevanne M, Monnier M, Cillard J, Delamarche A. Free radical scavenging and antioxidant effects of lactate ion: an in vitro study. Journal of applied physiology. 2000;89(1):169-75. 
43. Sundar SJ, Hsieh JK, Manjila S, Lathia JD, Sloan A. The role of cancer stem cells in glioblastoma. Neurosurgical focus. 2014;37(6):E6. doi:10.3171/2014.9.FOCUS14494. 44. Yasuda K, Onimaru R, Okamoto S, Shiga T, Katoh N, Tsuchiya K et al. [18F]fluoromisonidazole and a new PET system with semiconductor detectors and a depth of interaction system for intensity modulated radiation therapy for nasopharyngeal cancer. International journal of radiation oncology, biology, physics. 2013;85(1):142-7. doi:10.1016/j.ijrobp.2012.03.029.

45. Hatano T, Zhao S, Zhao Y, Nishijima K, Kuno N, Hanzawa H et al. Biological characteristics of intratumoral [F-18]fluoromisonidazole distribution in a rodent model of glioma. International journal of oncology. 2013;42(3):823-30. doi:10.3892/ijo.2013.1781. 
Table 1: Patients’ characteristics

\begin{tabular}{ll}
\hline Characteristic & Value \\
\hline Age (years) & \\
Mean SD & $63.5 \pm 14.8$ \\
Median & 66.0 \\
Range & $27-85$ \\
\hline Sex, $\mathrm{n}$ & \\
Male/Female & $17 / 15$ \\
\hline KPS & \\
Median & 75 \\
Range & $50-100$ \\
\hline EOR, \% & \\
Mean SD & $63.47 \pm 14.81$ \\
Median & 93.73 \\
Range & $0-100$ \\
\hline IDH1/2 mutations, $\mathrm{n}$ & \\
- & 24 \\
+ & 1 \\
Unknown & 7 \\
\hline
\end{tabular}

Abbreviations: KPS, Karnofsky performance status; EOR, extent of resection; IDH, isocitrate dehydrogenase. The IDH1/2 mutations were examined in 25/32 tumor samples, where $24 / 25$ cases were proved as de novo type of glioblastoma. 
Table 2: Optimal thresholds for each parameter

\begin{tabular}{|l|l|l|}
\hline & Threshold & $\chi 2$ statistic \\
\hline FMISO TNR & 4.19 & 1.91 \\
\hline FDG TNR & 1.42 & 2.84 \\
\hline GTV (ml) & 75.88 & 7.05 \\
\hline HV (ml) & 47.65 & 6.60 \\
\hline HMF(\%) & 39.00 & 1.24 \\
\hline hMTV (ml) & 15.01 & 9.46 \\
\hline hTLG (ml) & 49.41 & 10.9 \\
\hline age (y.o.) & 61 & 1.37 \\
\hline KPS & 70 & 0.208 \\
\hline EOR(\%) & 94.50 & 5.19 \\
\hline
\end{tabular}

Abbreviations: FMISO, fluoromisonidazole; TNR, tumor-to-normal region ratio; FDG, fluorodeoxyglucose; GTV, gross tumor volume; HV, hypoxia volume; HMF, hypermetabolic fraction of hypoxic volume; hMTV, metabolic tumor volume in hypoxia; hTLG, total lesion glycolysis in hypoxia; KPS, Karnofsky performance status; EOR, extent of resection. The optimal cutoff values generated maximum $\chi^{2}$ statistics for each parameter in categorizing patients by OS. 
Table3: Univariate analyses of patients' PFS and OS

\begin{tabular}{|c|c|c|c|c|}
\hline & \multicolumn{2}{|r|}{ PFS } & \multicolumn{2}{|r|}{ OS } \\
\hline & $\mathrm{p}$ value & HR & $\mathrm{p}$ value & HR \\
\hline FMISO TNR & 1.0 & $1.44(0.47-4.38)$ & 1.0 & $1.52(0.43-5.38)$ \\
\hline FDG TNR & 0.26 & $2.23(0.95-5.26)$ & 0.64 & $1.81(0.72-4.54)$ \\
\hline GTV (ml) & 1.00 & $1.18(0.27-5.24)$ & 0.14 & 3.49 (0.67-18.09) \\
\hline $\mathrm{HV}$ (ml) & 0.89 & $1.71(0.62-4.69)$ & 0.040 & 4.65 (1.53-13.19) \\
\hline HMF(\%) & 0.23 & 2.57 (0.97-6.77) & 0.22 & $2.67(0.99-7.21)$ \\
\hline hMTV (ml) & 0.0070 & $7.11(2.23-22.66)$ & 0.0028 & $19.93(3.90-101.96)$ \\
\hline hTLG (ml) & 0.040 & $3.86(1.45-10.27)$ & 0.037 & $4.28(1.51-12.15)$ \\
\hline age (y.o.) & 1.00 & $1.04(0.43-2.55)$ & 1.0 & $0.94(0.31-2.87)$ \\
\hline KPS & 0.50 & $1.89(0.79-4.49)$ & 0.34 & $2.82(0.88-5.36)$ \\
\hline EOR(\%) & 0.010 & $0.23(0.092-0.56)$ & 0.014 & $0.13(0.036-0.47)$ \\
\hline
\end{tabular}

Abbreviations: PFS, progression free survival; OS, overall survival; HR, hazard ratio; FMISO, fluoromisonidazole; TNR, tumor-to-normal region ratio; FDG, fluorodeoxyglucose; GTV, gross tumor volume; HV, hypoxia volume; HMF, hypermetabolic fraction of hypoxic volume; hMTV, metabolic tumor volume in hypoxia; hTLG, total lesion glycolysis in hypoxia; KPS, Karnofsky performance status; EOR, extent of resection. 
Table 4: Multivariate analyses of patients’ PFS and OS

\begin{tabular}{|c|c|c|c|c|c|c|c|c|}
\hline & \multicolumn{4}{|c|}{ Model 1 :PFS or OS HV+hMTV+EOR } & \multicolumn{4}{|c|}{ Model 2 : PFS or OS HV+hTLG+EOR } \\
\hline & \multicolumn{2}{|r|}{ PFS } & \multicolumn{2}{|c|}{ OS } & \multicolumn{2}{|r|}{ PFS } & \multicolumn{2}{|c|}{ OS } \\
\hline & $\mathrm{p}$ value & HR & $\mathrm{p}$ value & HR & $\mathrm{p}$ value & HR & $\mathrm{p}$ value & HR \\
\hline $\mathrm{HV}(\mathrm{ml})$ & 1.00 & $1.00(0.33-3.08)$ & 0.21 & $2.069(0.66-6.50)$ & 0.49 & $0.68(0.23-2.04)$ & 0.50 & $1.51(0.46-4.98)$ \\
\hline hMTV (ml) & 0.0020 & $6.68(2.01-22.24)$ & 0.0063 & $10.45(1.94-56.27)$ & & & & \\
\hline hTLG (ml) & & & & & 0.027 & $3.07(1.13-8.33)$ & 0.011 & $5.64(1.49-21.42)$ \\
\hline EOR(\%) & 0.0074 & $0.26(0.097-0.70)$ & 0.0042 & $0.12(0.029-0.52)$ & 0.0032 & $0.22(0.082-0.60)$ & 0.013 & $0.18(0.046-0.70)$ \\
\hline
\end{tabular}

Abbreviations: PFS, progression free survival; OS, overall survival; HR, hazard ratio; HV, hypoxia volume; hMTV, metabolic tumor volume in hypoxia; hTLG, total lesion glycolysis in hypoxia; EOR, extent of resection. To avoid co-linearity problem, Model 1 consisted of the hMTV + HV + EOR, and model 2 consisted of the hTLG + HV + EOR. 


\section{Figures legends}

Figure 1:

Lesion analysis of hypoxia glucose metabolism. (a)(b) FDG PET. (c) FMISO PET. (d) FLAIR MRI. (e) Gd enhanced T1-weighted MRI. Volume-of-interest was defined on FLAIR image (d). Blue area represents the high FDG uptake area (i.e., FDG TNR $\geq 1.0$ ) (a), green area represents the high FMISO uptake area (i.e., FMISO TNR $\geq 1.3$ ) (c), and red area represents with FDG-and-FMISO double-positive area (b). Gross total volumes were calculated from the VOIs which enclose the entire area showing gadolinium enhancement (e). Gray scale: SUV 0-5 for (a) and (b); SUV 0-2 for (c).

Figure 2:

Kaplan-Meier curves for patient's overall survival (OS). Patients with a higher hMTV had a significantly shorter OS (a). Patients with a higher hTLG had a significantly shorter OS (b). Patients with a higher EOR had a significantly longer OS (c). 
FDG

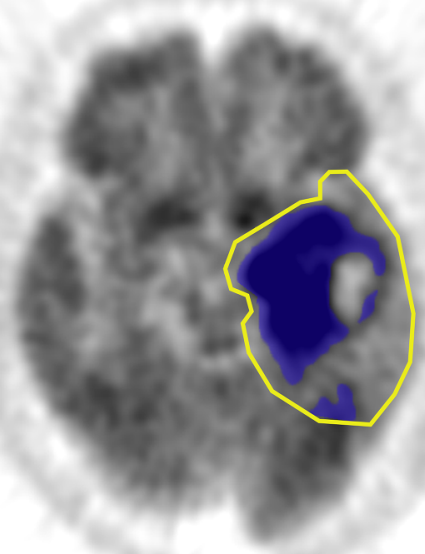

Fusion

FMISO

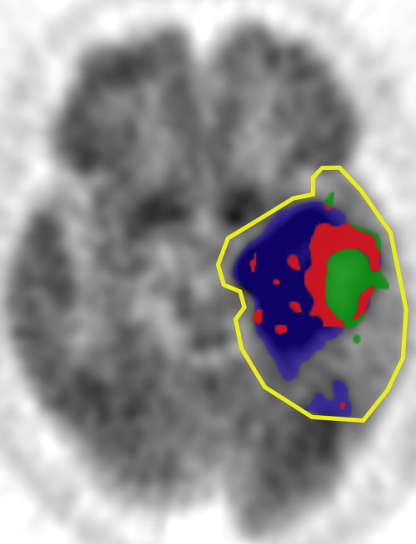

Figure 1

0.0

5.0

0.0

5.0

0.0

2.0

FLAIR

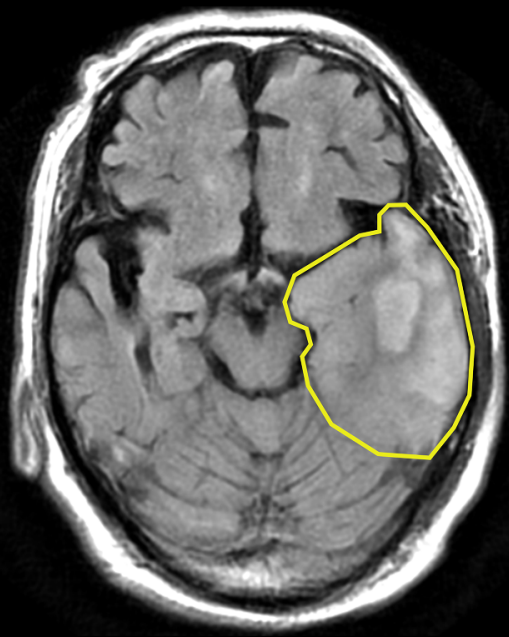

Gd-T1WI

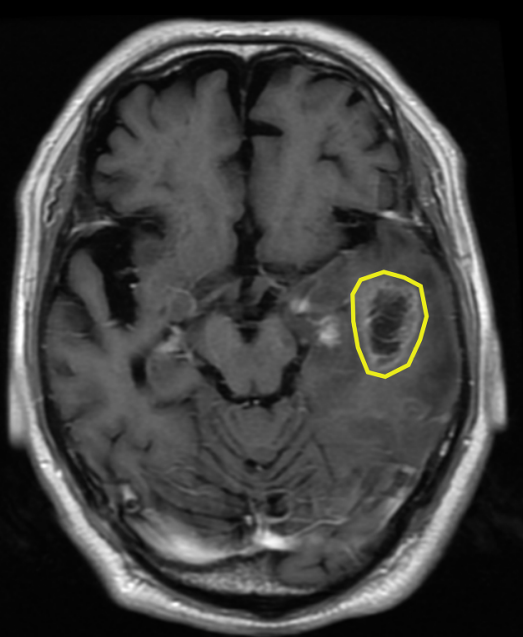


